International Journal of Child, Youth and Family Studies (2012) 4.1: 442-457

\title{
DEPRESSION AMONG CHINESE CHILDREN AND ADOLESCENTS: AREVIEW OF THE LITERATURE
}

\section{Maggie Zgambo, Fatch Kalembo, He Guoping, and Wang Honghong}

\begin{abstract}
The purpose of this review is to explore, identify, and discuss the predisposing factors and associated outcomes of depression in Chinese children and adolescents. For inclusion in the review, studies had to meet our objectives, be original peer-reviewed articles, conducted among Chinese children and adolescents in China. Articles were sourced through MEDLINE, EMBASE, Wan Fang Data, PsycINFO, and DOAJ databases. The results of the review indicate that the prevalence of depression symptoms in Chinese children and adolescents is high. The following factors are related to depression in Chinese children and adolescents: family, social factors, peer relations, gender, age, obesity, body image, and ethnicity. The outcomes of depression are poor academic performance, psychosocial retardation, conduct problems, cognitive distortion, and suicide. Depression is a major mental health problem among Chinese children and adolescents. This points to the need for longitudinally designed and controlled studies to establish effective preventive strategies.
\end{abstract}

Keywords: depression, Chinese, children, factors, outcomes

Maggie Zgambo is a graduate student in Nursing, Xiangya Medical College, Central South University, Tongzipo Road 172, Changsha 410013, China. (86) 187-17174954, Fax:

(86)731-82650268, E-mail: zgambo.maggie@yahoo.ca

Fatch Kalembo is a graduate student in Maternal Child and Adolescent Health, Tongji Medical College, Huazhong University of Science and Technology, Hangkong Road 13, Wuhan 430030, China. Phone: (86) 152-71811322, E-mail: kalembofatch@yahoo.com

He Guoping, Ph.D. (the corresponding author) is a Professor and Dean of Nursing Faculty, Xiangya Medical College, Central South University, Tongzipo Road 172, Changsha 410013, China. Phone: (86)731-82650268, Fax: (86)731-82650268, E-mail: hgpcsu@yahoo.com.cn

Wang Honghong, Ph.D. is a Professor and Head of Research Studies, Faculty of Nursing, Xiangya Medical College, Central South University, Tongzipo Road 172, Changsha 410013, China. Phone: (86)731-82650268, Fax: (86)731-82650268, E-mail:

honghong_wang@hotmail.com 
Globally, children have historically been thought not to be susceptible to depression. In recent years, this view has given way to an understanding that child depression not only exists, but also constitutes an insidious and major public health problem (Afifi, 2006). Depression is predicted to be the illness with the greatest negative impact and disease burden by the year 2020 (Lopez \& Murray, 1998). Major depression was the fourth most prevalent human disease in 1990 and is expected to rank second by the year 2020 (Andrews \& Szabo, 2002). Prior to the 1980s, depression was thought to be very rare in children and adolescents (Keenan \& Hipwell, 2005) but this view has changed in recent years. Its prevalence increases sharply with age precisely during adolescence. Although there is widespread acknowledgment that the onset of depression among children is still uncommon, estimates of the prevalence of major depression during adolescence range from 14\% to 20\% (Kessler, Avenevoli, \& Merikangas, 2001).

China has not been spared from incidents of depression in children and adolescents. Even though there is no official estimate of the prevalence of depression in the country, there are significant rates from studies on the subject. According to studies conducted in Taiwan, Nanjing/Hefei, and Beijing, prevalence of depression in children was found to be $10 \%$, 13.2\%, and 13.2\% respectively (Liu, 2003; Stewart \& Sun, 2007; Li et al., 2007). Studies done on adolescents in Hunan, Hong Kong, and Nanjing found that 22.9\%, 50\%, and 15.7\% of the participants were depressed respectively (Yang et al., 2010; Sun, Hui, \& Watkins, 2006; Hong et al., 2009). These rates are high taking into account that studies were conducted in schools. The rates are actually higher than those from Western countries (Chan, 2000; Sunita et al., 1999; Tepper et al., 2008; Sawyer et al., 2000). These high rates of depression in Chinese children and adolescents affect the lives of those afflicted in multiple areas of life and have been associated with a variety of factors. This paper examines peer-reviewed articles conducted on depression in China with an emphasis on predisposing factors and outcomes.

\section{Method}

We reviewed peer-researched articles done in China on depression in children and adolescents using the MEDLINE, DOAJ, WANFANG, PsycINFO, and Google Scholar databases. Some information was collected from a reliable source, namely the United Nations International Children's Emergency Fund (UNICEF). For inclusion in the review, studies had to be original peer-reviewed articles of any study design, conducted in China among Chinese children and adolescents aged 8 to 19 years. The search terms used in the electronic databases were: depression in children and adolescents in China; depression symptoms in children and adolescents; predisposing factors of depression symptoms; outcomes of depression symptoms in children and adolescents. The combined searches resulted in 1,834 articles. The articles were screened for eligibility by scanning the titles and 146 abstracts of the articles were 
retrieved for further assessment. The abstracts were further scrutinized, and 63 full text articles were obtained. Out of the 63 full text articles, only 45 met our inclusion criteria and were included in the review. The studies were excluded if they tackled depression among adults, were conducted outside China, studied subjects who were not Chinese children and adolescents, or if they were review articles. The results are presented under the following headings:

- $\quad$ Predisposing Factors,

- Associated Outcomes,

- $\quad$ The Way Forward

- Conclusion.

\section{Predisposing Factors}

Many predisposing factors have been associated with depression: family factors, social factors, age and gender, ethnicity and culture, and physical (or body) factors.

\section{Family factors}

As the core center for the development and life of children and adolescents, the Chinese family has been demonstrated to affect mental health status either positively or negatively. The economic, social, spiritual condition, liability condition, and status of the family have been shown to play a pivotal role in the mental health status of children and adolescents. Tactlessly, parents expose children to these factors through situations they feel are out of their control or out of ignorance, for instance, by leaving home or letting their children be raised by other people. It has been shown that children and adolescents who live without parents exhibit higher levels of depressive symptoms than those with parents around them (Wang, Yan, Hui, \& Juan, 2011).

In China, children and adolescents become victims of "without-parents” quandary due to migration, divorce, or death. For the past few years in her explosive economic growth, China has experienced the greatest migration of its citizens from rural areas to urban centers looking for greener pastures. This has resulted in a significant toll on the mental health of children left behind, particularly on children whose parents left early in their lives (Liu, Li, \& Ge, 2009). A cross-sectional study done in Anhui, Chongqing, and Guizhou indicated that the presence of parents in the early years of a child's life prevents depression in children. In the study, the Children's Depression Inventory (CDI) score was highest for children whose parents left before they reached the age of 3 years (mean $=43.97$; SD $=9.03$ ) compared with children whose parents left after they entered school (aged 7 years or older), who showed significantly fewer symptoms of depression. In addition, children whose parents went away before their third birthday had the most symptoms of trait anxiety (mean $=47.56$; $\mathrm{SD}=8.73$ ) (Liu et al., 2009). The presence of parents therefore appears to prevent depression symptoms in Chinese children and adolescents. 
Studies have also shown that the composition of the family exposes children to risk of depression symptoms. Children from divorced and single-parent families have been reported to have higher levels of anxiety and depression compared to children from nuclear and stem families (Dong Wang, \& Ollendick, 2002; Yu \& Seligman, 2002). The stress of single parenthood can lead to both paying less attention to a child and also to physical and verbal punishments that promote depression (especially when done frequently). Perceived parental warmth decreases the onset of depression symptoms in children and adolescents ( $\mathrm{Yu} \&$ Seligman, 2002; Greenberger, Chen, Tally, \& Dong, 2000). One Hong Kong study found the perception of a lack of parental understanding to be one of the strongest variables in predicting depressed moods in adolescents (Sunita et al., 1999). Some studies have indicated that lower family cohesion, higher family conflict, a poor quality of family relationships, and conflicts with parents lead to greater depression in children (Sun et al., 2006; Yu \& Seligman, 2002; Greenberger et al., 2000). Poor family cohesion in China increases the risk of depression in children and adolescents. Depression is decreased by higher levels of parental care and lower levels of parental indifference (Zhang, Li, \& Zou, 2011; Liu, 2003). Greenberger and colleagues (2000) stipulate that strong positive family relationships lessen the symptoms of depression.

\section{Social factors}

The social world impacts on the lives of Chinese children and adolescents positively or negatively. How young people relate with peers and teachers, what is expected in their peer groups, and social and academic achievements are elements that send messages affecting the mental status of the children and adolescents for good or for ill. There is evidence showing that perceptions of positive messages regarding children themselves, their world, and their future are negatively associated with depression, whereas a negative perception of the same messages has an opposite effect on depression symptoms (Liu, 2003). Negative messages are stressful. Stressful life events are negative life events. Negative life events are depressive (Yang et al., 2010; Greenberger et al., 2000). Negative life events predict higher levels of future depression symptoms (Yu \& Seligman, 2002). However, higher social and peer support lower the risk for depression. Peer support increases levels of self-esteem by moderating the effects of particular risk factors for depressive symptoms in each cultural setting (Yang et al., 2010; Sun et al., 2006; Greenberger et al., 2000). Being accepted by one's peers is very important for good mental health status in the life of every child and adolescent. Lack of peer acceptance and unpopularity predict symptoms of depression (Sunita et al., 1999; Li \& Zhang, 2008). A child who does not associate with peers could be lonely enough to be negatively affected in terms of mental health. Besides, it is evident that peer-related loneliness and seclusion are more predictive of depression than parent-related loneliness (Lau, Chan, \& Lau, 1999). Loneliness leads to depression. Children and adolescents who are lonely are most likely to develop symptoms of depression.

Teachers have also been known to cause stress in children and adolescents. 
Supportive teachers decrease the risk of depression symptoms in children by increasing levels of self-esteem. In one study done in Hong Kong, girls perceived higher levels of teacher support and lower levels of self-esteem (Sun et al., 2006; Yu \& Seligman, 2002). Teachers can erode or destroy the mental status and capacity of a child or adolescent through negative attitudes and unconstructive criticism. Children who have been harmfully disapproved of by teachers view criticism as an accusation or condemnation. Teachers ought to criticize children constructively. Ineffectual criticism is harmful to children and adolescents as they begin to have a negative attitude towards criticism and themselves. Perceived criticism directly influences the student depression (Lue, Wu, \& Yen, 2010). Positive criticism and good attitudes on the part of teachers increase levels of optimism and encourage sound coping skills. This strengthens the child to be spirited or resilient. Increasing levels of resilience, optimism, and positive affectivity (e.g., education) decrease levels of depressive symptoms in children and adolescents (Stewart \& Sun 2007; Zhang et al., 2011).

\section{Age and gender}

For a long time, depression has been known to be more common in girls than in boys. In China, the findings of many studies validate this notion, agreeing with a classification of adolescents (Lau et al., 1999; Sunita et al., 1999; Greenberger et al., 2000). Sex is directly related to expressions of depression with females being more susceptible than males (Lue et al., 2010; Hesketh, Ding, \& Jenkins, 2002). Tepper et al. (2008) argue that depressive symptoms do not differ between boys and girls but intensify with age. However, some authors have argued that gender differences in depressive symptoms are greater in some cultures than others. For instance, one comparative study done between adolescents in China and the United States found that gender differences in depressive symptoms were greater among the U.S. adolescents compared to the Chinese (Greenberger et al., 2000). One cross-sectional study found that the girls' depressive symptoms were more related to maternal messages while boys' depressive symptoms were more related to paternal messages (Liu, 2003).

\section{Ethnicity and culture}

The high prevalence of depression and the associated risk factors among adolescents are said by some researchers to be related to cultural factors (Zhang et al., 2011). Studies have noted differences in the prevalence and associated factors for adolescents' depression symptoms among various races and cultures. Other researchers reported that the occurrence of depression in adolescents might be associated with differences in race and cultural background (Park et al., 2010; Seaton, Caldwell, Sellers, \& Jackson, 2010). Auerbach, Eberhart, and Abela (2010) found that Chinese adolescents reported a higher level of depressive symptom than Canadian adolescents because of different cultures and beliefs. In Yu and Seligman's (2002) longitudinal study, 1,416 students ranging from 8 to 15 years of age were investigated using the Children Depression Inventory. It was found that Chinese children had significantly higher overall CDI scores than American children. 
These findings are similar to the findings from several studies in different parts of the world which indicate that depression levels and causative factors are not exactly the same among diverse racial and cultural groups (Franko \& Striegel-Moore, 2002; Bach \& Louw, 2010; Evans et al., 2008). By contrast, a two-year longitudinal study conducted on primary school children in Shanghai found the same mean depression scores as those in the West (Chen, Kenneth, \& Li, 1995). However, most studies agree there is a cultural difference. For example, one cross-sectional ethnocultural comparative study of children and adolescents was conducted in the Houston, Texas metropolitan area examining the following ethnic groups: Anglo or white; African or black; Native American; specific Hispanic groups including Cuban, Puerto Rican, Mexican and Central American; and specific Asian groups including Cambodian, Vietnamese, Indian, Pakistani, Chinese, Korean, and Pacific Islander. Subjects of Chinese descent were found to have the lowest prevalence of depression adjusted for impairment of 1.9\% (Roberts, Roberts, \& Chen, 1997).

Occurrence of depression in adolescents is associated with differences in race and cultural background and the conceptual organization of the symptoms characterizing depression are related to culture (Park et al., 2010; Seaton et al., 2010; Lu, Bond, Friedman, \& Chan, 2010). This suggests that measures for preventing and curing depression symptoms in children and adolescents should be culturally sensitive in any setting.

\section{Physical body factors}

Physical body fitness has been shown to have an effect on depression symptoms in Chinese children. Active children and adolescents have been proven to be less vulnerable to depression symptoms than those who are inactive. Regular physical exercise benefits children and adolescents with a positive enhancement in mood and lower rates of depression.

Researchers in China have come to conclude that body and mind exercises and freedom from physical maltreatment reduce the onset of depression and other psychopathologically-related problems in Chinese children and adolescents (Ying \& Liu, 2005; Hong et al., 2009; Jiang \& Zhu, 1997; Tao et al., 2006).

In one cross-sectional study, it was concluded that physical activity is inversely related to depression. It was found that participants who spent 1 to 7 hours per week, 8 to 14 hours per week, and more than 15 hours per week engaged in recreational physical activity, respectively, had odds ratios of $0.70(95 \% \mathrm{CI}=0.57,0.86), 0.68(95 \% \mathrm{CI}=0.53,0.88)$, and 0.66 (95\% CI $=0.50,0.87)$ for likelihood of being depressive, compared to their counterparts who spent 0 to 0.9 hours per week engaged in physical activity (Hong et al., 2009). Therefore, total inactivity or partial physical exercise in Chinese children and adolescents correlates with depression symptoms. Some authors have related depression to being overweight and having a poor body image (Tang et al., 2010; Li et al., 2007). The obese children with body dissatisfaction have significantly lower self-esteem and higher levels of depressive symptoms than the obese children without body dissatisfaction or children of normal weight (Shin \& Shin, 2008). There is a suggestion that the overweight perception is related to school-related stress and depression in both girls and boys (Bin et al., 2006). 


\section{Associated Outcomes}

Most of the outcomes of depression are unpleasant and disruptive. Many studies have found that depression symptoms have an impact on the lives of children and adolescents. Some of the depression outcomes have been discovered to affect depression symptoms and be caused by depression symptoms in some cases. For example, poor academic performance has been associated with depression symptoms and the same poor academic performance has been found to cause depression symptoms (Lue et al., 2010; Greenberger et al., 2000; Chen \& Li, 2000; Hesketh et al., 2002; Li \& Zhang, 2008). It could be that persistent poor academic performance causes depression due to feelings of failure resulting in low self-esteem. Symptoms of depression have a negative impact on school performance (UNICEF, 2004). The characteristics of depression, such as aggressive behavior, withdrawal, lack of interest, and social problems, can all distract a child from concentrating on education (Liu et al., 2000). In a related study, grades in school were found to be affected by depression (Greenberger et al., 2000). This was replicated in a cross-sectional study done among 12-year-old Chinese children where depression was found to be associated with academic performance in that children who had poor academic performance had more depressive symptoms than those with good academic performance (Lue et al., 2010). This means that the more depressive symptoms are present the greater the likelihood of poor school performance. Depression symptoms are preventable, avoidable, and curable, and given that children and adolescents will compose the world of tomorrow, it is perhaps not overstating the case to say the future of the present world depends on their academic achievement. Therefore children and adolescents who have symptoms of depression should be helped to overcome this condition and unleash their potential to realize their educational goals.

The effects of depression symptoms not only affect the child or adolescent but also his or her parents, teachers, peers, and friends. A number of studies have positively associated symptoms of depression with conduct problems, cognitive distortion, aggressive, disruptive, or antisocial behavior, poor peer relations, and low social competency in Chinese children (Chen \& Li, 2000; Liu, Tein, Sandler, \& Zhao, 2005; Lue et al., 2010; Chen et al., 1995; Liu et al., 2000; UNICEF 2004). Depression is the main culprit of antisocial behavior and poor social competence. A child who is depressed fails to socialize effectively. Social incompetence is social retardation. A child who cannot socialize is disposed to low self-esteem, feelings of failure, and is often alone. This creates a vicious circle of depressive symptoms as some outcomes are predisposing factors too (e.g., loneliness). Depression symptoms have a tendency of reoccurrence and it is therefore important to curb initial predisposing factors. Social competence is important in the life of a child to facilitate socialization for maturity and peer matching. Socialization develops social and psychological skills. Depressed moods are a significant phenomenon in the social development of Chinese children. Depression symptoms have a deleterious impact on future achievement for the reason that poor social and psychological development, adjustment to difficulties and 
emotional status underlie mental health problems (Chen \& Li, 2000; Lau et al., 1999; Zhang et al., 2011).

As perilously as depression symptoms have been neglected in China, effects are prominent including the sundry suicide cases of children and adolescents. Many studies of Chinese children and adolescents have found depression to be the major cause of suicide incidents or ideation (Phillips et al., 2002; Liu et al., 2005; Sun et al., 2006). It is evident that China has a high national suicide rate of two to three times the global average with 300,000 suicides annually. This accounts for $30 \%$ of the world's suicides with the majority being females in the age group of 15 to 24 (Samuel \& Pozi, 2008; Yip, Liu, Hu, \& Song, 2005; UNICEF, 2004). Adolescence is a stage of life in transit from childhood to adulthood. Depression during this period is very common in many individuals. This happens due to hormonal imbalances, an individual fighting to attain independence and seeking social approval and identity from peers which, when failed or compromised, results in self-hatred, rejection, poor social competence, poor coping skills, poor life skills, and so depression develops. Since this period of development happens to everybody, effective strategies to prevent predisposing factors leading to depression symptoms in this age group are inconsistent and neglected by policy-makers, teachers, health workers and even parents. These factors are neglected because they are regarded as normal. An escalation of problems resulting from depression at this stage of life is what calls attention to the condition. Adolescents' concomitant problems range from failed first romantic associations, early sex involvement, unwanted pregnancy, contracting sexually transmitted infections like HIV/AIDS, substance abuse, and alcoholism which can lead to depression putting these children and adolescents at risk of suicide.

There is a need to develop strategies that will meet children's and adolescents' emotional needs and bolster their psychological status in homes, schools and hospitals. Chinese children and adolescents should be taught knowledge of life skills, coping mechanisms and the power of resilience. Let there be knowledge dissemination that will inform children and adolescents of the need to seek assistance when confronted with a psychological problem. Normally in China, traditional societies often view the need for counseling as indicating severe mental illness (UNICEF, 2004) yet minor mental illness when untreated may gradually develop into severe conditions. This suggests the need to educate every group of persons that come in contact with children and adolescents including parents, guardians, teachers and health workers to start prioritizing the mental health status of children and adolescents. Furthermore, there is a need for more mental health workers and more dedication because suicide ideation and incidence among Chinese children and adolescents is high. In studies done in China, suicide ideation is highly rated ranging from 16\% to 32\% (Hesketh et al., 2002; Liu, 2004; Sun et al., 2006; Chan, 1995; Yip et al., 2004). 


\section{The Way Forward and Conclusion}

This review provides a better and more comprehensive understanding of the importance and intensity of problems associated with depression symptoms in Chinese children and adolescents. It also highlights the significance of preventing depression symptoms in children and adolescents. The fact that rates of depression in children and adolescents are so high means policy-makers should pay attention to, internalize, and implement guidelines to prevent and manage depression. There is a need to educate parents, guardians, and teachers on the severity of depression symptoms (as a mental disease), predisposing factors, their preventability and their devastating effects on the lives of children and adolescents. This will help these adults to have an overall understanding of depression in children and adolescents, hence assisting in its prevention and in pursuing mental health assistance when necessary.

Mental health services are lacking in most of the programs in China serving adolescents and children. We therefore recommend the integration of mental health services into youth-friendly programs and school curricula by government or non-governmental organizations (NGOs). This will help to equip adolescents and children with life skills to help them to effectively cope with problems that result from lack of peer, teacher and family support, low self-esteem, and negative life events. At the same time, we recommend more youth-friendly services, programs, projects, and centers in China. This will not only help in reaching out many children and adolescents but also provide a rare opportunity for adolescents to practice physical and intellectual exercises that help to develop their mental health. These centers will also assist in identifying adolescents and children with symptoms of depression and suicidal ideation and provide counseling services to help them deal with their problems.

Our review has also identified poor family relationships, lack of parental care due to divorce or migration of parents from rural to urban areas as some of the factors predisposing adolescents and children to depression. We therefore recommend that the government introduce more counseling centers closer to people so that parents with marital problems and affected children can receive counseling support. The government should also come up with policies that promote the development and creation of job opportunities for people in the rural areas to help prevent migration to urban areas. There is limited psychosocial support offered to Chinese adolescents and children with depression. This highlights the need for culturally tailored public health studies to come up with depression models of care that encourage peer psychosocial support for adolescents and children. Most of the studies conducted in China on depression in adolescents and children were found to be cross-sectionally designed; we therefore call for more longitudinal studies like cohorts and quasi experiments to be conducted which can determine scientifically reliable preventive strategies of depression symptoms in children and adolescents. Such study methods could determine culturally sensitive and effective interventions and approaches towards reducing and eradicating 
International Journal of Child, Youth and Family Studies (2012) 4.1: 442-457

predisposing factors of depression symptoms in children and adolescents. There are also some research gaps in the area of knowledge, attitude, practices, and beliefs of teachers, health workers, parents, children, and adolescents on depression symptoms, which are the basis for mapping intervention protocols.

Depression is a major mental health problem for Chinese children and adolescents which requires the collaboration of all stakeholders involved with the care of adolescents and children in planning and implementing programs that will help to overcome the problem. Adolescents and children should be involved from the outset of such programs. Government and non-governmental organizations should provide financial muscle to support the programs in order to ensure their sustainability. 


\section{References}

Afifi M. (2006). Positive health practices and depressive symptoms among high school adolescents in Oman. Singapore Medical Journal, 47(11), 960-966.

Andrews, G., \& Szabo, M. (2002). Preventing major depression in young people. British Journal of Psychiatry, 181, 460-462.

Auerbach, R. P., Eberhart, N. K., \& Abela, J. R. Z. (2010). Cognitive vulnerability to depression in Canadian and Chinese adolescents. Journal of Abnormal Child Psychology, 38(1), 57-68.

Bach, J. M., \& Louw, D. (2010). Depression and exposure to violence among Venda and Northern Sotho adolescents in South Africa. African Journal of Psychiatry, 13(1), 25-35.

Bin, X., Ping, C. C., Spruijt-Metz D., Reynolds, K., Palmer, P. H., Gallaher, P., et al. (2006). Weight perception, academic performance, and psychological factors in Chinese adolescents. American Journal of Health Behavior, 30(2), 115-124. doi: http://dx.doi.org/10.5993/AJHB.30.2.1

Chan, D. (1995). Reasons for living among Chinese adolescents in Hong Kong. Suicide and Life-Threatening behavior, 25(3): 347-358. doi: 10.1111/j.1943-278X.1995.tb00957.x

Chan, D. W. (2000). Depressive symptoms and coping strategies among Chinese adolescents in Hong Kong. Journal of Youth Adolescence, 24(3), 267-279.

Chen, X., Kenneth, R. H., \& Li, B. S. (1995). Depressed mood in Chinese children: Relations with school performance and family environment. Journal of Consultant Clinical Psychology, 63(6), 938-947.

Chen, X., \& Li, B. S. (2000). Depressed mood in Chinese children: Development significance for social and school adjustment. International Journal of Behavioral Development, 24(4), 472-479.

Dong, Q., Wang, Y. P., \& Ollendick, T. H. (2002). Consequences of divorce on the adjustment of children in China. Journal of Clinical Child Psychology, 31(1),101-110.

Evans, D., Buxton, D. C., Borisov, A., Manatunga, A. K., Ngodup, D., \& Raison, C. L. (2008). 
International Journal of Child, Youth and Family Studies (2012) 4.1: 442-457

Shattered Shangri-la: Differences in depressive and anxiety symptoms in students born in Tibet compared to Tibetan students born in exile. Social psychology and Psychiatric Epidemiology, 43(7), 429-436.

Franko, D. L., \& Striegel-Moore, R. H. (2002). The role of body dissatisfaction as a risk factor for depression in adolescent girls. Are the differences black and white? Journal of Psychosomatic Research, 53(5), 975-983.

Greenberger, E., Chen, C., Tally, S. R., \& Dong, Q. (2000). Family, peer, and individual correlates of depressive symptomatology among U.S. and Chinese adolescents. Journal of Consulting and Clinical Psychology, 68(2), 209-219. doi: 10.1037/0022-006X.68.2.209

Hesketh, T., Ding, Q. J., \& Jenkins, R. (2002). Suicide ideation in Chinese adolescents. Social Psychology and Psychiatric Epidemiology, 37(5), 230-235.

Hong, X., Li, J. Q., Xu, F., Tse, Lap A., Liang, Y. Q., Wang, Z. Y., et al. (2009). Physical activity inversely associated with the presence of depression among urban adolescents in regional China. BMC Public Health, 9(1), 148. doi:10.1186/1471-2458-9-148

Jiang, B., \& Zhu, B. (1997). The mental health of college and middle-school students in Shanghai and its relationship with physical exercises. Psychological Sciences, 1(1), 3-9.

Keenan, K., \& Hipwell, E. A. (2005). Preadolescent clues to understanding depression in girls. Clinical Child and Family Psychology Review, 8(2), 89-105.

Kessler, R. C., Avenevoli, S., \& Merikangas, K. R. (2001). Mood disorders in children and adolescents: An epidemiologic perspective. Biological Psychiatry, 49(12), 1002-1014.

Lau, S., Chan, D. W. K., \& Lau, P. S. Y. (1999). Facets of loneliness and depression among Chinese children and adolescents. Journal of Social Psychology, 13(6), 713-729.

Li, H. J., \& Zhang, Y. (2008). Factors predicting rural Chinese adolescents' anxieties, fears and depression. School Psychology International, 29(3), 376-384.

Li, Y. P., Ma, G. S., Schouten, E. G., Hu, X. Q., Cui, Z. H., Wang, D., \& Kok, F. J. (2007). 
International Journal of Child, Youth and Family Studies (2012) 4.1: 442-457

Report on childhood obesity in China (5) body weight, body dissatisfaction, and depression symptoms of Chinese children aged 9-10 years. Biomedical and Environmental Sciences, 20(1), 11-18.

Liu, X. (2004). Sleep and adolescent suicidal behavior. SLEEP, 27(7), 1351-1358.

Liu, X. C., Guo, C., Okawa, M., Zhai, J., Li, Y., Uchinyama, M., et al. (2000). Behavioral and emotional problems in Chinese children of divorced parents. Journal of American Academy of Child \& Adolescent Psychiatry, 39(7), 896-903.

Liu, X. C., Tein, J. Y., Sandler, I. N., \& Zhao, Z. T. (2005). Psychopathology associated with suicide attempts among rural adolescents of China. Suicide and Life-Threatening Behavior, 35(3), 265-276.

Liu, Y. L. (2003). Parent-child interaction and children's depression: The relationships between parent-child interaction and children's depressive symptoms in Taiwan. Journal of Adolescence, 26(4), 447-457

Liu, Z., Li, X., \& Ge, X. (2009). Left too early: The effects of age at separation from parents on Chinese rural children's symptoms of anxiety and depression. American Journal of Public Health, 99(11), 2049-2054.

Lopez, A. D., \& Murray C. C. (1998). The global burden of disease, 1990-2020. Nature Medicine, 4(11), 1241-1243.

Lu, A., Bond, M. H., Friedman, M., \& Chan, C. (2010). Understanding cultural influences on depression by analyzing a measure of its constituent symptoms. International Journal of Psychological Studies, 2(1), 55-70.

Lue, B. H., Wu, W. C., \& Yen, L. L. (2010). Expressed emotion and its relationship to adolescent depression and antisocial behavior in Northern Taiwan. Journal of Formosan Medical Association, 109(2), 128-137.

Park, M. H., Kim, T. S., Yim, H. W., Jeong, S. H., Lee, C., Lee, C. U., et al. (2010). Clinical characteristics of depressed patients with a history of suicide attempts: Results from the CRESCEND study in South Korea. Journal of Nervous and Mental Disease, 198(10), 748-754.

Phillips, M. R., Yang, G., Zhang, Y., Wang, L., Ji, H., \& Zhou, M. (2002). Risk factors for 
International Journal of Child, Youth and Family Studies (2012) 4.1: 442-457

suicide in China: A national case-control psychological autopsy study. The Lancet, 360(9347), 1728-1736.

Roberts, R. E., Roberts, C. R., \& Chen Y. R. (1997). Ethno-cultural differences in prevalence of adolescent depression. American Journal of Community Psychology, 25(1), 95-110.

Samuel, L., \& Pozi, L. (2008). Suicide in China: Unique demographic patterns and relationship to depressive disorder. Current Psychiatry Reports, 10(1), 80-86. doi: $\underline{10.1007 / s 11920-008-0014-5}$

Sawyer, M., Arney, F. M., Baghurst, P. A., Clark, J. J., Graetz, B. W., Kosky, R. J., et al. (2000). The mental health of young people in Australia (Child and adolescent component of the national survey of mental health and well-being). Canberra: Mental Health and Special Programs Branch, Commonwealth Department of Health and Aged Care.

Seaton, E. K., Caldwell, C. H., Sellers, R. M., \& Jackson, J. S. (2010). An intersectional approach for understanding perceived discrimination and psychological well-being among African American and Caribbean black youth. Developmental Psychology, 46(5), 1372-1379.

Shin, N. Y., \& Shin, M. S. (2008). Body dissatisfaction, self-esteem, and depression in obese Korean children. Journal of Pediatrics, 152(4), 502-506.

Stewart, D., \& Sun, J. (2007). Resilience, depression in children: Mental health promotion in primary schools in China. International Journal of Mental Health Promotion, 9(4), 37-46.

Sun, R. C. F., Hui, E. K. P., \& Watkins, D. (2006). Towards a model of suicidal ideation for Hong Kong Chinese adolescents. Journal of Adolescence, 29(2), 209-224.

Sunita, M., Stewart, C. L., Betson, H., Lam, S. F., Chung, H. H., \& Ho, T. C. F. (1999). The correlates of depressed mood in adolescents in Hong Kong. Journal of adolescent health, 25(1), 27-34. doi: http://dx.doi.org/10.1016/S1054-139X(98)00018-4

Tang, J., Yu, Y., Du, Y., Ma, Y., Zhu, H., \& Liu, Z. (2010). Association between actual weight status, perceived weight and depressive, anxious symptoms in Chinese adolescents: A cross-sectional study. BMC Public Health, 10(1), 594. 
International Journal of Child, Youth and Family Studies (2012) 4.1: 442-457

Tao, F. B, Huang, K., Kim, S., Ye, Q., Sun, Y., Zhang, C. Y., et al. (2006). Correlation between psychopathological symptoms, coping style in adolescent and childhood repeated physical, emotional maltreatment. Chinese Journal of Pediatrics Hua Er Ke Za Zhi, 44(9), 688-693.

Tepper, P., Liu, X., Guo, C., Zhai, J., Liu, T., \& Li, C. (2008). Depressive symptoms in Chinese children and adolescents: Parent, teacher, and self-reports. Journal of Affective Disorders, 111(2-3), 291-298.

United Nations International Children's Emergency Fund (UNICEF). (2004). The Children: Adolescents. UNICEF/China accessed in January 2012 from http://www.unicef.org/china/children_877.htm.l

Wang, Y. J., Yan, H., Hui, F. L., \& Juan L. H. (2011). Preliminary study on the health status among the left behind children in the Xian Tao rural area of Hubei province. Zhongguo Dang Dai Er Ke Za Zhi [Chinese Journal of Contemporary Pediatrics], 13(12), 977-980.

Yang, J., Yao, S., Zhu, S., Zhang, C., Ling, Y., Abela, J. R., et al. (2010). The impact of stress on depressive symptoms is moderated by social support in Chinese adolescents with subthreshold depression: A multi-wave longitudinal study. Journal of Affective Disorders, 127(1-3), 113-121.

Ying, H., \& Liu, J. I. (2005). The study of the effects of physical exercise volume on the depressive level and psychological mediator (body-esteem) in college students. Journal of Tianjin Institute of Physical Education, 20(1), 728-736.

Yip, P. S. F., Liu, K. Y., Hu, J., \& Song, X. M. (2005). Suicide rates in China during a decade of rapid social changes. Social Psychiatry and Psychiatric Epidemiology, 40(10), 792-798. doi: 10.1007/s00127-005-0952-8

Yip, P. S. F., Liu, K. Y., Lam, T. H., Stewart, S. M., Chen, E., \& Fan, S. (2004). Suicidality among high school students in Hong Kong, SAR. Suicide and Life Threatening Behavior, 34(3), 284-297.

Yu, D. L., \& Seligman, M. (2002). Preventing depressive symptoms in Chinese children. Prevention and Treatment, 5(9), 1-39.

Zhang, Y, Li, H., \& Zou, S. (2011). Association between cognitive distortion, type d 
International Journal of Child, Youth and Family Studies (2012) 4.1: 442-457

personality, family environment, and depression in Chinese adolescents. Depression Research and Treatment, 2011(143045), 1-8. 